\title{
Improving the image quality of photoacoustic tomography (PAT) by using a negative acoustic lens
}

Changhui Li, Geng Ku, Lihong V. Wang

Changhui Li, Geng Ku, Lihong V. Wang, "Improving the image quality of photoacoustic tomography (PAT) by using a negative acoustic lens," Proc. SPIE 6856, Photons Plus Ultrasound: Imaging and Sensing 2008: The Ninth Conference on Biomedical Thermoacoustics, Optoacoustics, and Acoustooptics, 685623 (28 February 2008); doi: 10.1117/12.763533 


\title{
Improving the image quality of photoacoustic tomography (PAT) by using a negative acoustic lens
}

\author{
Changhui Li, Geng Ku, and Lihong V. Wang \\ Optical Imaging Laboratory, Department of Biomedical Engineering \\ Washington University in St. Louis, St. Louis, MO, 63130
}

\begin{abstract}
Although a small point ultrasound transducer has a wide acceptance angle, its signal-to-noise (SNR) is low due to the high thermal-noise-induced electric voltages in the transducer, which is a result of its small active area. By contrast, a finite size flat transducer has high sensitivity (good SNR), but the acceptance angle is generally small, which limits its application in reconstruction-based photoacoustic tomography (PAT). In this paper, we report a negative lens concept to increase the acceptance angle for a flat transducer. We also provide phantom experiments that demonstrate this concept can greatly increase the detection region for PAT and without losing sensitivity.
\end{abstract}

Keywords: photoacoustic tomography, negative lens, directivity

\section{INTRODUCTION}

Photoacoustic (PA) tomography (PAT) acquires biomedical images by detecting ultrasound signals generated by tissues after they absorb the radiation energy of a light pulse. ${ }^{1,2}$ This imaging modality is non-invasive, and it combines sensitive optical contrast and high acoustic resolution. PAT has been successfully applied in imaging both small animals and human tissues. ${ }^{3-5}$ Among many kinds of ultrasound detectors used for PAT, ${ }^{4,6-8}$ single finite size flat transducers are widely used due to its high sensitivity and simplicity, and it has achieved good images in PAT. ${ }^{4,9}$

However, single finite size flat transducers generally have very limited angles of acceptance. From the principle of reciprocity, the radiation pattern of a transducer also describes the acceptance pattern of the transducer. Theoretically, the radiation pattern of the wave amplitude in the far field of a round shaped transducer with a radius of $a$ can be described approximately by a circular baffled piston with directivity factor given as ${ }^{10}$

$$
d(\theta)=2\left|\frac{J_{1}(k a \sin (\theta))}{k a \sin (\theta)}\right|,
$$

where $J_{1}$ is the Bessel function of the first kind, $k=2 \pi / \lambda$ and $\lambda$ is the wavelength, and $\theta$ is the deflected angle relative to the normal direction of the transducer surface. For example, Fig. 1 shows the directivity factor of a $1 / 4$ " diameter and $2.25 \mathrm{MHz}$ center frequency transducer immersed in water. In this figure, radiation power decreases over $-30 \mathrm{~dB}$ when the radiation angle is larger than 4 degrees, which demonstrates the small acceptance angle for the flat transducer.

Only signals coming within the acceptance angle can be effectively detected, and thus the acceptance angle determines the detection region in PAT. As shown in Fig. 2, the light grey area represents the PA active region, which contains the PA sources illuminated by the laser. Although a single flat transducer scans the whole activated region along the dashed circular trajectory and inwardly faces the scanning center, due to the limitation of the transducer acceptance angle, only PA signals generated within the middle darker region can be detected with certainty, and that region defines the field of view (FOV) for the detecting system.

PA sources outside the FOV may not be fully reconstructed due to possible signal losses. To increase the area of the FOV, the distance between the flat transducer and the PA source must be increased, which results

Further author information: (Send correspondence to Changhui Li) Changhui Li: E-mail: cli@biomed.wustl.edu, Telephone: 13149359586

Photons Plus Ultrasound: Imaging and Sensing 2008: The Ninth Conference on Biomedical Thermoacoustics, Optoacoustics, and Acousto-optics, edited by Alexander A. Oraevsky, Lihong V. Wang, Proc. of SPIE Vol. 6856, 685623, (2008) - 1605-7422/08/\$18 - doi: 10.1117/12.763533 


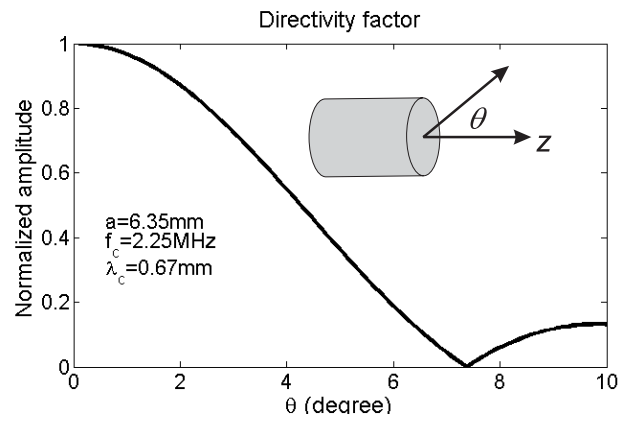

Figure 1. The directivity factor of a flat round shape transducer with a radius of $6.35 \mathrm{~mm}$. The transducer has a center frequency of $2.25 \mathrm{MHz}$ and its corresponding center wavelength in water is $0.67 \mathrm{~mm}$. The normal direction of the transducer surface is $\hat{z}$.

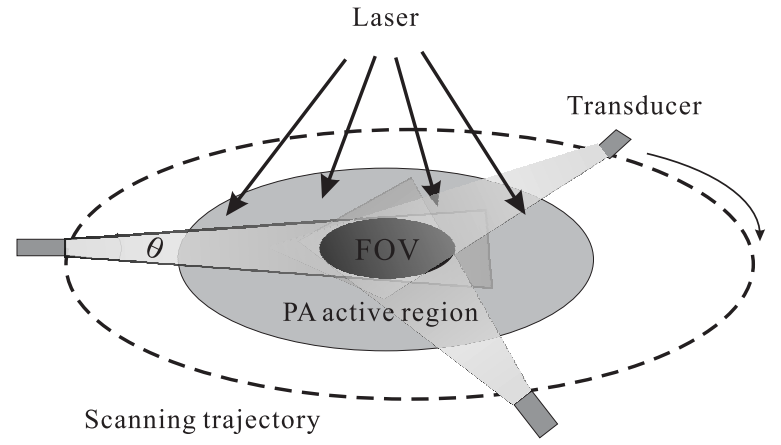

Figure 2. Photoacoustic active region and the field of view (FOV). A single flat transducer scans the PA source region along a circular trajectory. The transducer is always facing directly to the center of scanning region. The light grey area (PA active region) contains the PA sources which absorb the illuminating laser. The field of view corresponds to the overlap area of all detection areas, the area limited by the marked acceptance angle $(\theta)$, along scanning positions.

in drawbacks such as decreased signal strength and inconvenience in some applications. In the next section, we will present a new method by introducing a negative lens concept to enlarge the acceptance angles of single flat transducers. In the third section, we also provide phantom experiments to demonstrate this method. Finally, we give a conclusion in the last section.

\section{NEGATIVE LENS CONCEPT}

\subsection{Theoretical Basis}

From the principle of reciprocity, a larger detection angle for the flat transducer means that the ultrasound emitted from a flat transducer can be diverged into a larger radiation angle. In optics, a light beam can be diverged by a concave lens (negative lens). The mechanism of light divergence by a negative lens is based on the fact that the speed of light in the lens is slower than in air. By the same idea, an ultrasound wave can also be diverged by the acoustic counterpart - an acoustic negative lens.

The ultrasound in PAT generally propagates in the medium, such as the soft tissue, at a speed similar or equal to its speed in water $(1.5 \mathrm{~mm} / \mu \mathrm{s})$. The lens is made of solids, which generally have higher acoustic speed than water, so an acoustic negative lens has a convex shape as shown in Fig. 3. By adding an acoustic negative lens over the flat transducer surface, the emitted acoustic wave can be diverged. The divergence of the emitted acoustic wave can be approximately described by Snell's law:

$$
\frac{\sin (\theta)}{v}=\frac{\sin \left(\theta^{\prime}\right)}{v^{\prime}}
$$

Thus, if $v^{\prime}>v$, we have $\theta^{\prime}>\theta$, and the wave will be diverged. Snell's law is accurate only when the wavelength is much smaller than the size of the lens, which may not always be the case. For example, the lens used in this 


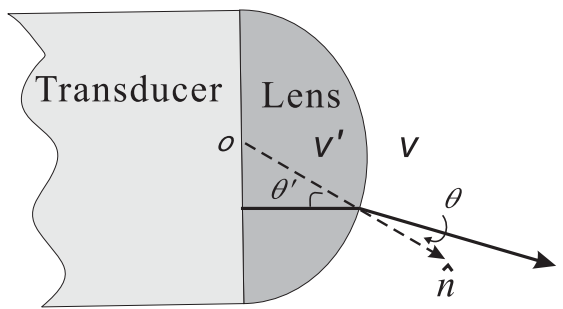

Figure 3. Geometry of an acoustic negative lens. $v^{\prime}$ and $v$ are sound speeds in the lens and surrounding medium, respectively. The divergent angle depends on the sound speed, as well as the geometry shape of the lens.

paper has a $1 / 2$ " diameter and is made of acrylic plastic. The center frequency of the transducer is $2.25 \mathrm{MHz}$, the center wavelength inside the lens is $1.22 \mathrm{~mm}$, which is about one-tenth of the diameter of the lens, so Snell's law can not be used for accurate calculation. This case requires the study of the wave diffraction, which is crucial for the image reconstruction, and we will discuss it in subsection 2.3 .

\subsection{Making The Negative Lens}

The lens material, acrylic plastic, has an acoustic speed of $2.75 \mathrm{~mm} / \mu \mathrm{s}$ and density of $1.19 \mathrm{~g} / \mathrm{cm}^{3}$. The lens was made by cutting a rod with a diameter of $1 / 2$ " and height of $1 / 2$ " in half along its center axis. Then it was epoxied to the surface of the transducer as shown in Fig. 4. The transducer has a 1/4" active area and thus the negative lens completely covers the active area. Later, we also painted the lens and part of the transducer white by silica gel to diminish the effect of direct laser illumination onto the transducer

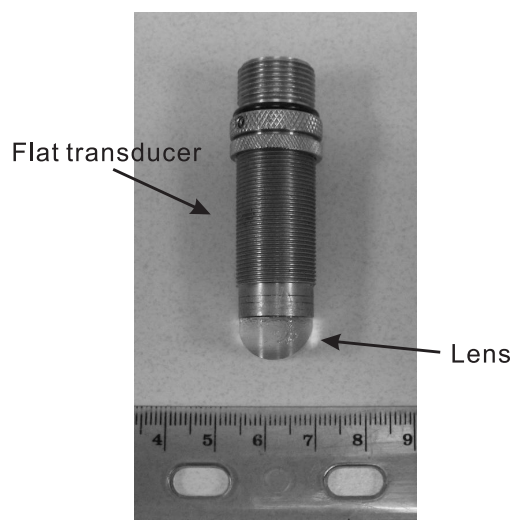

Figure 4. A negative lens made from the acrylic plastic is glued to the surface of a transducer. .

\subsection{Modified Reconstruction Algorithm}

Although the negative lens diverges the emitted ultrasound waves, the divergence is not uniform. Thus, the directivity factor for a flat transducer with a negative lens will be different with Eq. (1). Beside the radiation pattern, due to the differences in acoustic speeds between the lens and surrounding medium, as well as the shape of the lens, the arriving time (time-delay) of signals from the same distance but different incoming angles will be different too. Both effects must be carefully considered in the reconstruction algorithm. For simplicity, we assume that the new directivity factor, $d_{n e g}(\theta)$, is independent of the wavelength, and we also introduce the time domain counterpart factor, $t_{d}(\theta)$, to describe the changes in signal time-delays.

Before we discuss the reconstruction algorithm used for the transducer with a negative lens, we would first present the one for the flat transducer. Based on the experiment setup in Fig. 6, the reconstruction algorithm used for the flat transducer in this paper is the $2 \mathrm{D}$ version of the reconstruction formula from: ${ }^{11}$

$$
p_{0}^{(b)}(\mathbf{r})=\sum_{i}\left[2 p\left(\mathbf{r}_{i}, t\right)-2 t \frac{\partial p\left(\mathbf{r}_{i}, t\right)}{\partial t}\right] \frac{\cos (\theta)}{\left|\mathbf{r}-\mathbf{r}_{\mathbf{i}}\right|^{2}}
$$


where $p_{0}^{(b)}(\mathbf{r})$ is the reconstructed value at $\mathbf{r}, p\left(\mathbf{r}_{i}, t\right)$ is the signal received at location $\mathbf{r}_{\mathbf{i}}$ and at $t=\left|\mathbf{r}-\mathbf{r}_{\mathbf{i}}\right| / c$ ( $c$ is the acoustic speed in the water), $\cos (\theta)=\hat{n}_{i}^{s} \cdot\left(\mathbf{r}-\mathbf{r}_{\mathbf{i}}\right) /\left|\mathbf{r}-\mathbf{r}_{\mathbf{i}}\right|$ is the cosine of the relative angle between the normal direction of the transducer surface with respect to reconstructed point at $\mathbf{r}$, and $i$ is the index of the locations during the scanning.

With a negative lens, we assume the radius of the lens is $R$ and the acoustic speed in the lens is $c^{\prime}$. By modifying Eq. (3), we obtained the corresponding reconstruction algorithm with the negative lens as

$$
p_{0}^{(b)}(\mathbf{r})=\sum_{i}\left[2 p\left(\mathbf{r}_{i}, t^{\prime}\right)-2 t^{\prime} \frac{\partial p\left(\mathbf{r}_{i}, t^{\prime}\right)}{\partial t^{\prime}}\right] \frac{\cos (\theta)}{d_{n e g}(\theta)\left|\mathbf{r}-\mathbf{r}_{\mathbf{i}}\right|^{2}}
$$

where $t^{\prime}$ is now modified by

$$
t^{\prime}=t_{d}(\theta)\left[\frac{\left|\mathbf{r}-\mathbf{r}_{\mathbf{i}}\right|-R}{c}+\frac{R}{c^{\prime}}\right]
$$

Thus the modified version gives the compensation of the amplitude change and considers the time delay differences.

In this preliminary study of a negative lens in PAI, the new directivity factor $d_{\text {neg }}(\theta)$ and time delay factor $t_{d}(\theta)$ were obtained empirically from both simulation and trial and error. The simulation was based on the pseudo-spectral time-domain (PSTD) ${ }^{12}$ method. In the simulation, a short ultrasound pulse (with temporal width the same as the period of the center frequency) generated by the transducer passes through the negative lens. A normalized wave amplitude of the simulated wave front in the far field is shown in Fig. 5. By studying the simulated wave front and comparing reconstructed images with objects, we empirically obtained

$$
\begin{aligned}
d_{\text {neg }}(\theta) & \approx 10^{-\theta} \\
t_{d}(\theta) & \approx \frac{1}{1+0.03 \theta}
\end{aligned}
$$

where $\theta$ is in radian.

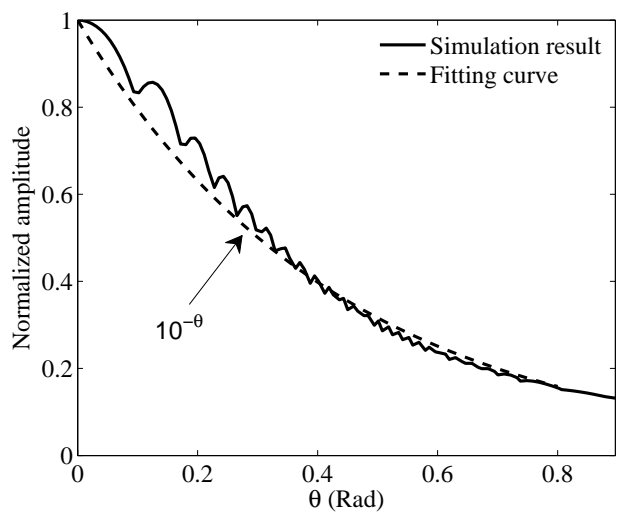

Figure 5. A snapshot of the acoustic wave in the time-domain of a pulse propagating after passing the negative lens. The negative lens boundary is marked by the white dashed line.

\section{EXPERIMENT}

\subsection{Phantom Experiments}

Phantom experiments were done in PAT to demonstrate the power of the negative lens concept. As shown in Fig. 6 , the PA source contained six black human hair rings glued on top of optical fibers, and the interval between hair samples was about $2 \mathrm{~cm}$. A Nd:YAG laser (LOTIS TII model LS-2137/2) generates 10-15 ns $532 \mathrm{~nm}$ laser pulses with a repetition rate of $10 \mathrm{~Hz}$, which were diverged by a combination of a concave lens and a ground 
glass, so that all six hair rings were illuminated. Two identical transducers with a $2.25 \mathrm{MHz}$ central frequency and a 0.25 inch diameter active area (Krautkramer 113-822-300, ISS $2.25 \times 0.25 \mathrm{NF}$ ) were used. One transducer had a negative lens as shown in Fig. 4. Both the phantom and the transducer were immersed in a tank filled with water. The transducer evenly scanned the object along a horizontal circle with a radius of about $8.0 \mathrm{~cm}$, stopping at 240 points. PA signals were recorded by a Gage card (Gage Applied, Inc., CS14100), and were then used for image reconstruction later.

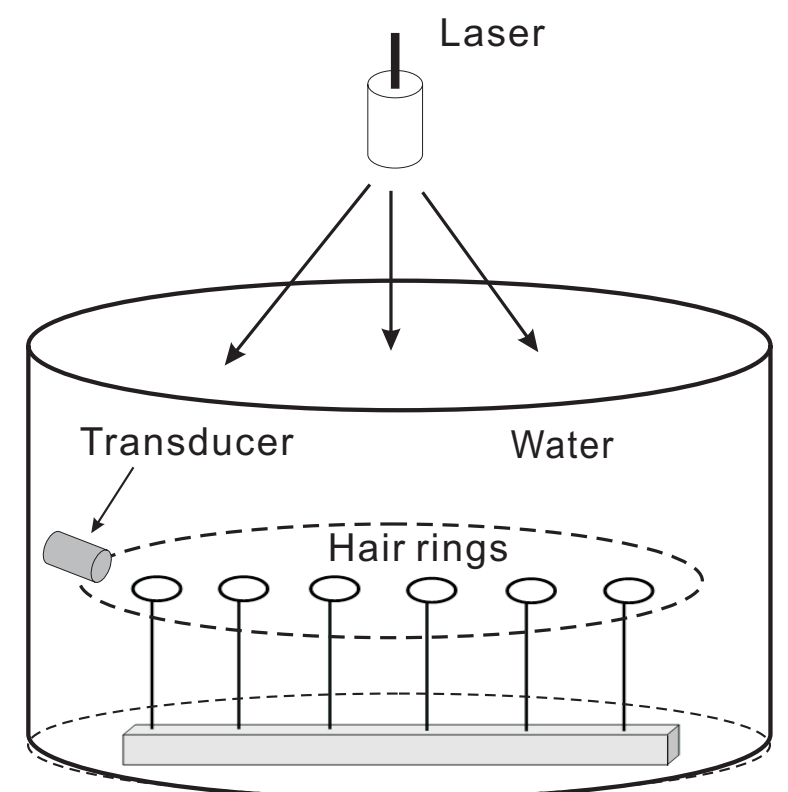

Figure 6. Experimental setup. The phantom contains six human hair rings, which were glued to the top of optical fibers of same length. The fibers were vertically fixed on a acrylic plastic base, and both the fiber and acrylic base do not absorb laser. Transducers, with or without a negative lens, scan the hair phantom along a circular path and at the same depth as the phantom. Both phantom and transducers were immersed in a tank filled with water. The laser beam is diverged so that all six hair rings can be illuminated.

Two experiments were done, one used the flat transducer without the negative lens and another one used the negative lens. The reconstructed images based on Eq. (3 and 4) are show in Fig. 7.

Significant differences exist between the two results. Without the negative lens, as shown in Fig. 7(a), only the hair sample close to the scanning center are correctly reconstructed, and only vertical parts are prominently reconstructed for the rest five hair rings. On the contrary for the result in Fig. 7(b), where the data is gathered by another flat transducer with negative lens, all six hair rings are successfully reconstructed. Apparently, negative lens greatly improved the quality of the PAT images.

\subsection{Discussions of the Result}

It can be explained by the differences in FOV between those two experiments. Because the generated PA signals prefer to propagate perpendicularly to the boundary ${ }^{13} \mathrm{PA}$ signals generated from hair rings propagate radially outward. As shown in Fig. 8, a hair ring is placed off center and on the $\hat{x}$ axis. The ultrasound signals generated by this hair ring are marked as short arrows. Since the signals generated by the left and right parts of the ring propagate close to $\pm \hat{x}$ direction, thus transducers, whether with or without a negative lens, can detect them at positions around " 1 " and "2" due to the small incident angle. However, the top and bottom side of the ring generate signals traveling close to $\pm \hat{y}$ direction, the incident angles reaching the transducer are determined by the location of the ring and radius of the scanning circle, and the angle increases with the distance away from the center. As shown in the figure, $\theta$ represent the incident angle. When the hair ring moves away from certain distance, where the incident angle is larger than the acceptance angle of the flat transducer, the flat transducer can not detecte the signals and thus the top and bottom part of the hair ring can not be reconstructed. On 
Flat transducer

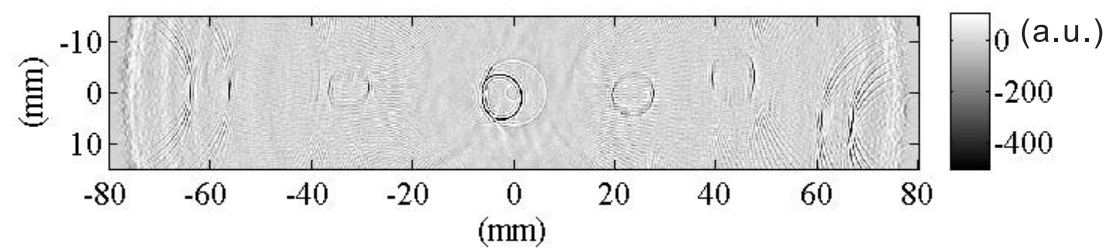

(a)

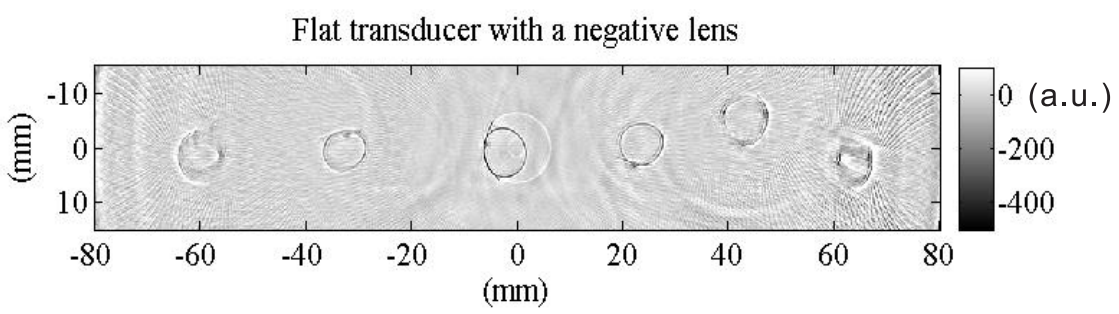

(b)

Figure 7. Reconstructed images for two experiments. (a) The reconstructed image from data gathered by a flat transducer without the negative lens; (b) The reconstructed image from data gathered by another same kind of flat transducer with a negative lens.

other words, the hair ring moves out of the FOV of the flat transducer. However, the larger acceptance angle of the transducer with a negative lens allows it to detect signals from top and bottom parts of the hair rings in a wider range than the flat transducer does, and so the images of those parts of rings can be reconstructed. From this experiment, the negative lens significantly increases the FOV.

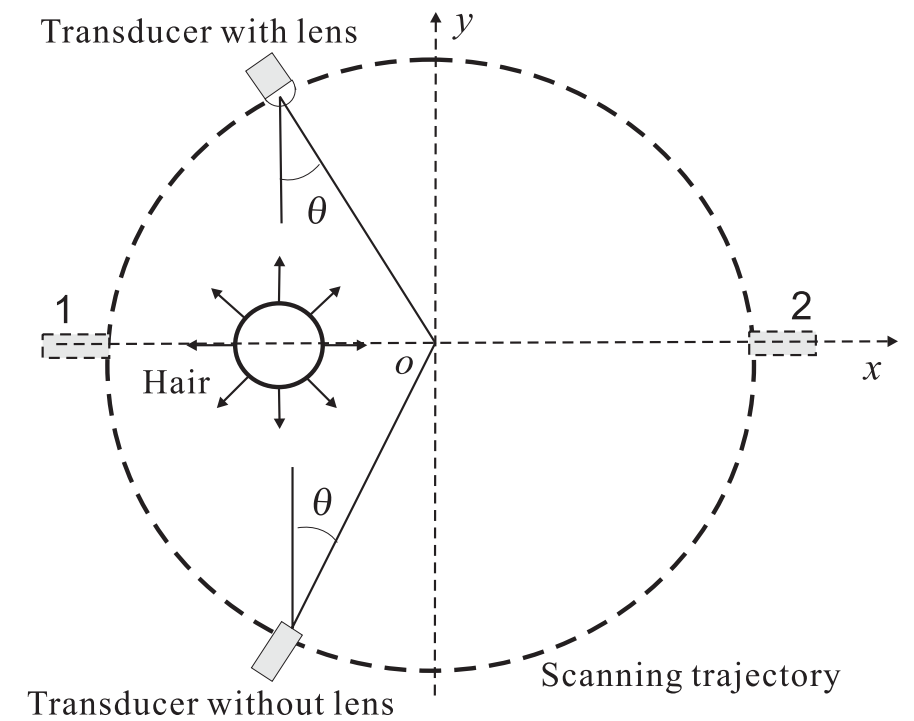

Figure 8. Two hairs are placed along $x$-axis with one vertically aligned and another horizontally aligned. PA signals generated by these two hairs traveling perpendicular to the hair boundary. Transducers, with or without negative lens, face the center and scan the region along a circular path. $\theta$ represents the incoming angle of the signals from the horizontal hair.

Although the image by using the negative lens has advantages over that from the flat transducer, the image boundaries are not sharp. There are several possible reasons:

1. The shape or material of the lens has defects; 
2. The estimated directivity factor and time-delay factor are not accurate;

3. Internal ultrasound reflections at the boundary between the lens and the surrounding medium lead to unwanted signals.

4. Impedance mismatch at the outer boundary of the lens reflects part of the signal, which decreases the strength of the received signal.

Even the first two problems can be solved by advanced techniques and theoretical studies. Where the last two can be minimized by acoustic coating, or could be avoided if we can find such materials with impendence match to make the lens. An alternative idea is to remove the negative lens, and replace the flat transducer by a convexly shaped surface. In this way, the transducer is a "negative focused transducer", and we expect this kind of transducer could perform better in PAT.

\section{CONCLUSIONS}

Both theoretical analysis and phantom experiment demonstrate that the negative lens significantly improves over the single flat transducer in PAT. This idea can also be used in the microwave-induced thermoacoustic tomography, where the laser is replaced by the microwave source. Furthermore, the shortcomings of the negative lens can be overcome by replacing it by a "negative focused transducer".

\section{Acknowledgment}

This project was sponsored in part by National Institutes of Health Grant Nos. R01 NS46214 and R01 EB000712. 


\section{REFERENCES}

1. M. H. Xu and L. H. V. Wang, "Photoacoustic imaging in biomedicine," Review of Scientific Instruments $\mathbf{7 7}(4), 2006$.

2. L. V. Wang and H.-i. Wu, Biomedical Optics : Principles and Imaging, Wiley, Hoboken, N.J., 2007.

3. C. G. A. Hoelen, F. F. M. de Mul, R. Pongers, and A. Dekker, "Three-dimensional photoacoustic imaging of blood vessels in tissue," Optics Letters 23(8), pp. 648-650, 1998.

4. X. D. Wang, Y. J. Pang, G. Ku, X. Y. Xie, G. Stoica, and L. H. V. Wang, "Noninvasive laser-induced photoacoustic tomography for structural and functional in vivo imaging of the brain," Nature Biotechnology 21(7), pp. 803-806, 2003.

5. R. I. Siphanto, K. K. Thumma, R. G. M. Kolkman, T. G. van Leeuwen, F. F. M. de Mul, J. W. van Neck, L. N. A. van Adrichem, and W. Steenbergen, "Serial noninvasive photoacoustic imaging of neovascularization in tumor angiogenesis," Opt. Express 13(1), pp. 89-95, 2005.

6. I. O. Wygant, X. Zhuang, P. S. Kuo, D. T. Yeh, O. Oralkan, and B. T. Khuri-Yakub, "Photoacoustic imaging using a two-dimensional cmut array," in Ultrasonics Symposium, 2005 IEEE, X. Zhuang, ed., 4, pp. 1921-1924, 2005.

7. J. J. Niederhauser, M. Jaeger, R. Lemor, P. Weber, and M. Frenz, "Combined ultrasound and optoacoustic system for real-time high-contrast vascular imaging in vivo," IEEE Transactions on Medical Imaging 24(4), pp. 436-440, 2005.

8. P. C. Beard, F. Perennes, and T. N. Mills, "Transduction mechanisms of the fabry-perot polymer film sensing concept for wideband ultrasound detection," IEEE Transactions on Ultrasonics Ferroelectrics and Frequency Control 46(6), pp. 1575-1582, 1999.

9. X. Wang, D. L. Chamberland, and D. A. Jamadar, "Noninvasive photoacoustic tomography of human peripheral joints toward diagnosis of inflammatory arthritis," Opt. Lett. 32(20), pp. 3002-3004, 2007.

10. A. D. Pierce, Acoustics: An Introduction to Its Physical Principles and Applications, Acoustical Society of America, New York, 1989 ed edition ed., 1989.

11. M. H. Xu and L. H. V. Wang, "Universal back-projection algorithm for photoacoustic computed tomography," Physical Review E 71(1), p. 016706, 2005. Part 2.

12. Q. H. Liu, "Large-scale simulations of electromagnetic and acoustic measurements using the pseudospectral time-domain (pstd) algorithm," IEEE Transactions on Geoscience and Remote Sensing 37(2), pp. 917-926, 1999. Part 2.

13. Y. Xu, L. V. Wang, G. Ambartsoumian, and P. Kuchment, "Reconstructions in limited-view thermoacoustic tomography," Medical Physics 31(4), pp. 724-733, 2004. 